\title{
SYNTHETIC HR DIAGRAMS FOR LUMINOUS STARS : A TEST OF STELLAR EVOLUTION THEORY
}

\author{
EMMA NASI \\ Osservatorio Astronomico \\ Padova Italy
}

The composite HR diagram for luminous stars in our galaxy by Humphreys and McElroy(1984) is the only source which provides enough data for the comparison with the results of massive stars theoretical models. The luminosity limit for completeness of Humphreys' and McElroy's cata$\log (1984)$ is about $\mathrm{M}_{\mathrm{Bol}}=-8 \mathrm{mag}$, when restricted to $3 \mathrm{Kpc}$ from the Sun. Inside these limits there are 378 luminous stars in galactic associations and clusters, whose distances are known with sufficient reliability . The correspondent observational HR diagram is reproduced in Fig. 1 . Synthetic HR diagrams for massive stars were constructed with the aim of reproducing the observed features for galactic luminous stars.

By means of a Montecarlo technique, stars were randomly distributed in the $\mathrm{M}_{\mathrm{Bol}}$-Log $\mathrm{T}_{\text {eff }} \mathrm{plane}$, weighted on the Salpeter's initial mass function and with a constant star formation rate. The different evolutionary scenarios considered for the comparison with observations are :

a) Evolution with mass loss by stellar wind all across the HR diagram as for Case C by Maeder (1981a,b) shown in Fig 2 ;

b) Evolution with mass loss by stellar wind and convective overshooting from the central cores as for $\lambda=1$ by Bressan et al.(1981) in Fig 3 ;

c) Evolution with mass loss, convective overshooting and modified CNO opacity as in Bertelli et al. (1984) in Fig 4

The number of stars in Figures 2,3 and 4 is the same as in Figure 1 . The comparison of the relative number of stars in different regions of the observed HR diagram with the distribution expected from models with mass loss (Fig 2) and with mass loss and overshooting (Fig 3) evidences an excess of A type stars and a main sequence theoretical width not sufficient to account for the observed distribution. As discussed in Bertelli et al.(1984), if on the observational ground the catalog incompleteness is the more serious problem, theoretical models give results in good agreement with the observed distribution only with a suitable combination of mass loss, overshooting and a moderate increase of standard radiative opacities in the region of the $\mathrm{CNO}$ ionization. The synthetic HR diagram in Fig 4 shows the best agreement with the observed one(Fig 1) , thus supporting the idea that standard theory must be modified. A modification of the opacities is actually one way to improve the agreement between theory and observations . 


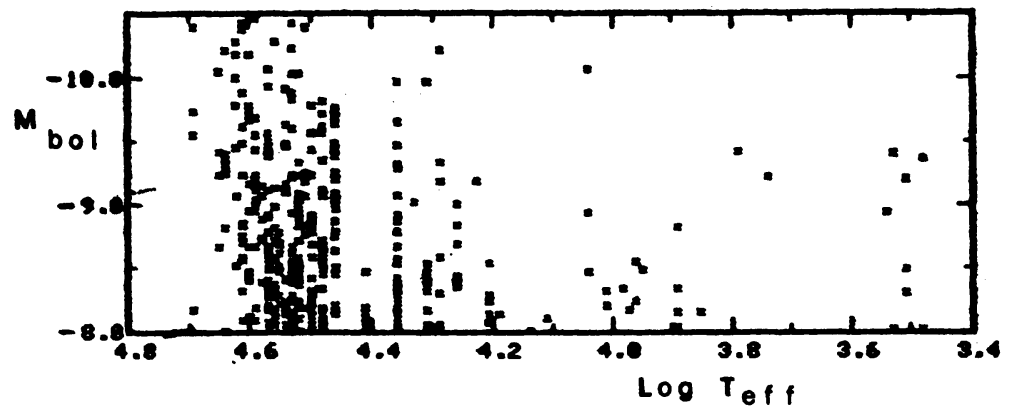

Fig. 1

Fig. 2
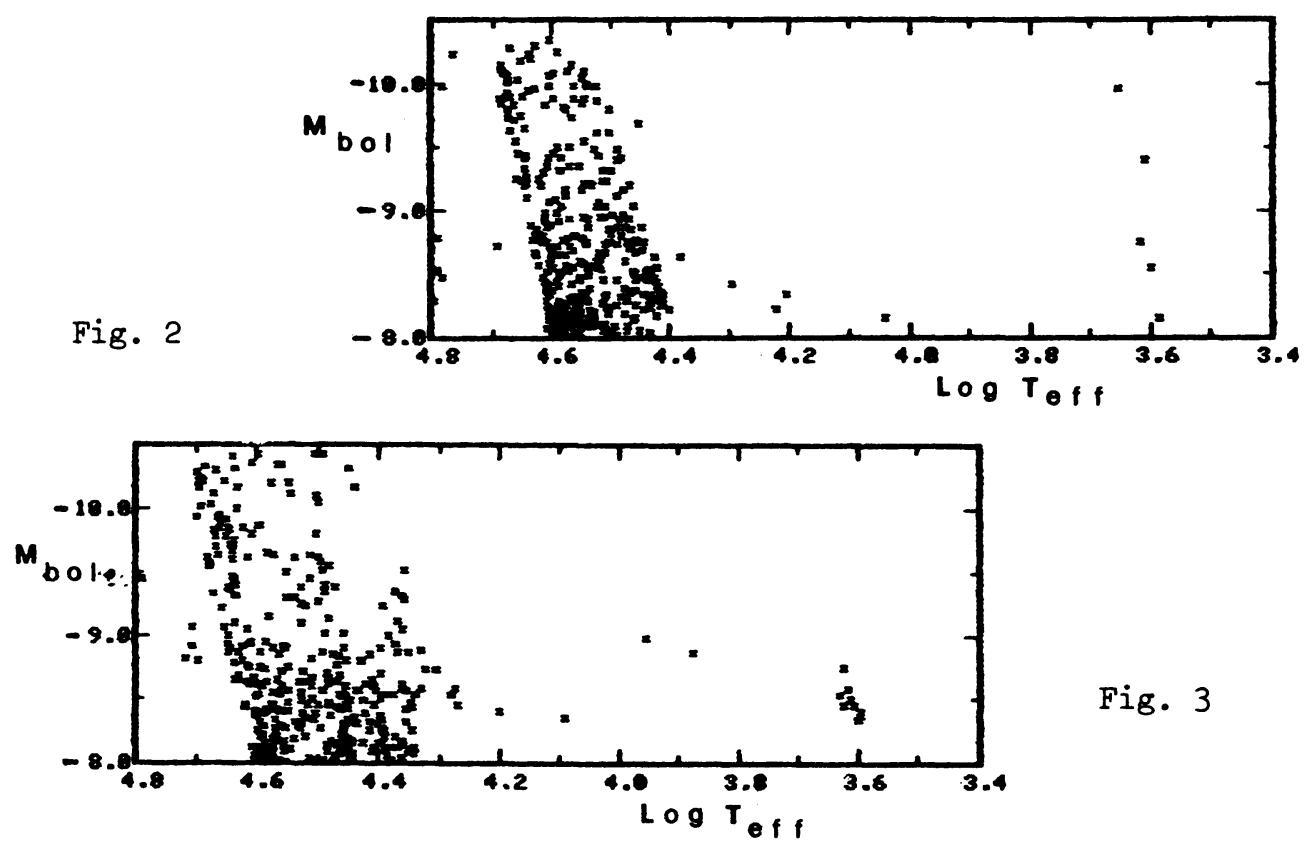

Fig. 3

Fig. 4

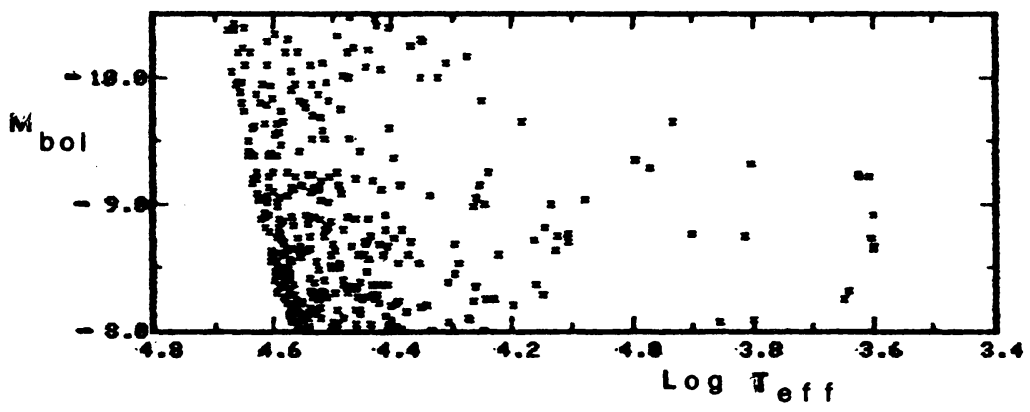

REFERENCES

Bertelli,G.,Bressan,A.G.and Chiosi,C. : 1984,Astron.Astrophys. 130 ,279 Bressan,A.G.,Bertelli,G.and Chiosi,C. :1981,Astron.Astrophys. 102 , 25 Humphreys, R.M.and McElroy,D.B. : 1984,Astrophys.J. 284,565

Maeder,A.:1981a, Astron. Astrophys. 99,97

Maeder,A. : 1981b, Astron. Astrophys. 102,401 